\title{
LETTER \\ Arrangement of Scattering Points in Jakes' Model for i.i.d. Time-Varying MIMO Fading
}

\author{
Hiroshi NISHIMOTO $^{\dagger a)}$, Student Member, Toshihiko NISHIMURA ${ }^{\dagger b)}$, Takeo OHGANE ${ }^{\dagger c)}$, \\ and Yasutaka OGAWA ${ }^{\dagger \mathrm{d})}$, Members
}

\begin{abstract}
SUMMARY For simulating i.i.d. time-varying MIMO channels using multiple Jakes' rings, it is desirable to generate channels having stable statistics with fewer scatterers. The statistical property of the conventional Jakes' model may depend on the initial phase set assigned to scattering points. In this letter, we present simple and effective conditions on arrangement of scattering points to achieve stable fading properties. The results show that the proposed arrangement provides higher statistical stability in generating time-varying channels.

key words: MIMO, time-varying Rayleigh fading, Jakes' model, scattering points
\end{abstract}

\section{Introduction}

Recently, high data-rate service with high mobility has been one of the growing demands for future wireless communications. The multiple-input multiple-output (MIMO) system [1]-[3] is already the core technology for some standards to achieve such high data speeds [4]. Thus, there are many opportunities to use a time-varying MIMO channel model in performance evaluations of MIMO systems.

Jakes' model has been extensively used for simulating time-varying Rayleigh fading with U-shaped Doppler power spectrum [5], [6]. The model can be simply applied to MIMO channels. When each element of a MIMO channel matrix independently obeys the model, i.e., by using multiple scattering rings, we can obtain independent and identically distributed (i.i.d.) time-varying MIMO channels, theoretically. Statistical validity of the MIMO channels is achieved with a sufficiently large number of scatterers in the rings. Since it is not desirable for prompt numerical analyses to locate many scattering points, relatively small numbers of scatterers are often used in fact. However, decreasing the scattering points without consideration on their arrangement may lead statistics fluctuation depending on the initial phase at each point, as will be shown later. In this letter, we establish simple and effective conditions on arrangement of scattering points in MIMO Jakes' model for sufficient stability of statistics.

\footnotetext{
Manuscript received April 4, 2007.

Manuscript revised July 5, 2007.

${ }^{\dagger}$ The authors are with the Graduate School of Information Science and Technology, Hokkaido University, Sapporo-shi, 0600814 Japan.

a)E-mail: hn@ist.hokudai.ac.jp

b) E-mail: nishim@ist.hokudai.ac.jp

c)E-mail: ohgane@ist.hokudai.ac.jp

d)E-mail: ogawa@ist.hokudai.ac.jp

DOI: 10.1093/ietcom/e90-b.11.3311
}

\section{Simplified Jakes' Model}

We consider a narrow-band MIMO system equipped with $N_{\text {tx }}$ transmit (TX) antennas and $N_{\text {rx }}$ receive (RX) antennas. It is assumed that the $k$ th $\mathrm{RX}$ antenna, which is surrounded by a scattering ring $R_{k l}$ with $M$ scattering points for paths from the $l$ th TX antenna, moves with a velocity $v$ as illustrated in Fig. 1. Thus, a time-varying channel $h_{k l}(t)$ from the $l$ th TX antenna to the $k$ th RX antenna, which is an element of the $k$ th row and $l$ th column in the MIMO channel matrix, can be generated using the Jakes' model with the corresponding scattering ring $R_{k l}$.

We define $x$ - and $y$-axes as the moving direction and its orthogonal one, respectively. In a complex baseband system, the channel $h_{k l}(t)$ is represented as

$$
\begin{aligned}
h_{k l}(t) & =\sum_{m=1}^{M} a_{k l, m} e^{j\left\{2 \pi f_{D}\left(\cos \theta_{k l, m}\right) t+\phi_{k l, m}\right\}} \\
& =\sum_{m=1}^{M} h_{k l, m}(t),
\end{aligned}
$$

where $a_{k l, m}, \theta_{k l, m}$, and $\phi_{k l, m}$ are a received amplitude, an angle of arrival, and an initial phase of the $m$ th scattered wave component $h_{k l, m}(t)$, respectively, and $f_{D}$ is the maximum Doppler frequency. The Doppler shift caused by the $m$ th scatterer is $f_{D} \cos \theta_{k l, m}$. Both $\theta_{k l, m}$ and $-\theta_{k l, m}$ contribute to the same Doppler shift because of $\cos \theta_{k l, m}=\cos \left(-\theta_{k l, m}\right)$. Thus, in Jakes' model, it is known that scattering points

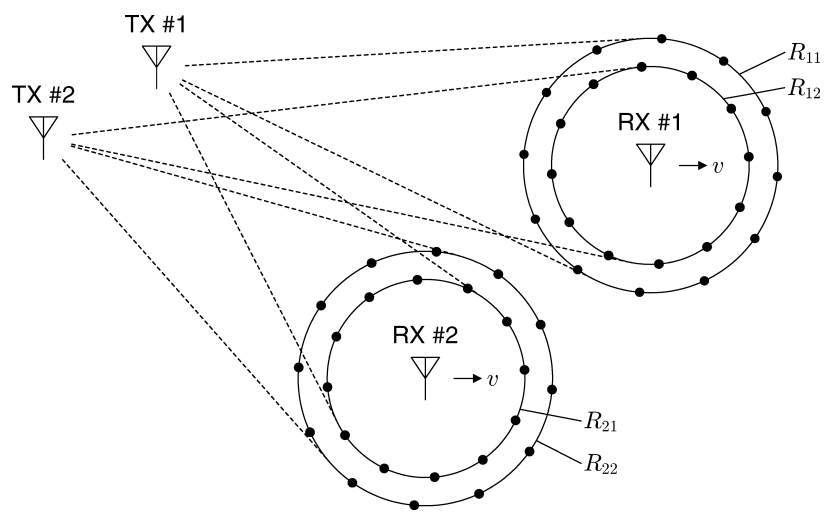

Fig. 1 Concept of an i.i.d. time-varying MIMO channel model using multiple Jakes' rings $\left(N_{\mathrm{tx}}=N_{\mathrm{rx}}=2\right)$. 


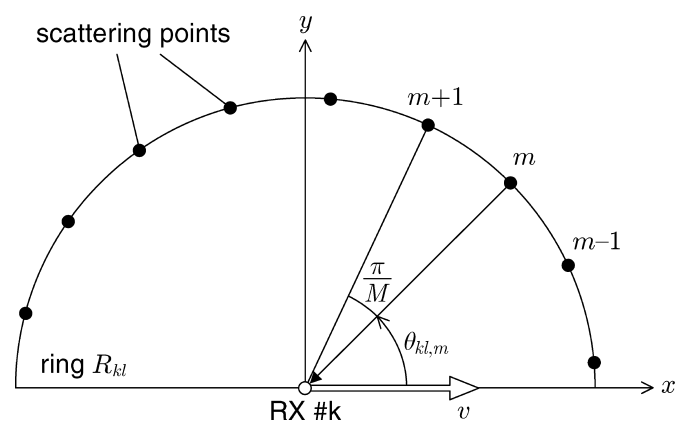

Fig. 2 Simplified Jakes' model.

should not be arranged symmetrically to the $x$-axis.

Here, we define that all $M$ points are distributed only in the range of $0 \leq \theta_{k l, m} \leq \pi$ with equal interval $\pi / M$ to facilitate the following discussion, as illustrated in Fig. 2. Although, strictly speaking, each ring becomes a semicircle under the above angular condition, we still refer to it as ring hereinafter. In addition, we assume $a_{k l, m}=a=1 / \sqrt{M}$ for all $k, l$, and $m$ for the sake of simplicity. Under the assumption, we obtain an ergodic channel power $E\left[\left|h_{k l}(t)\right|^{2}\right]=1$.

\section{Intra-Ring Condition}

First, we consider the scattering ring $R_{k l}$ only. When an arbitrary pair of scattering points $m$ and $m^{\prime}$ locates in $y$-axis symmetry, i.e., $\theta_{k l, m}=\pi-\theta_{k l, m^{\prime}}$, their absolute values of Doppler shift components are the same, i.e., $\left|f_{D} \cos \theta_{k l, m}\right|=$ $\left|f_{D} \cos \left(\pi-\theta_{k l, m^{\prime}}\right)\right|$. In this case, the channel may have exceptional characteristics depending on the initial phase. For example, let us consider the case of $\phi_{k l, m}=\phi_{k l, m^{\prime}}=$ 0 . A superposed wave composed of these two scattered waves is expressed as $a e^{j 2 \pi f_{D}\left(\cos \theta_{k l, m}\right) t}+a e^{-j 2 \pi f_{D}\left(\cos \theta_{k l, m}\right) t}=$ $2 a \cos \left\{2 \pi f_{D}\left(\cos \theta_{k l, m}\right) t\right\}$. This means that this component does not have its imaginary part, and that the amplitude becomes double. For another example, in the case of $\phi_{k l, m}=0$ and $\phi_{k l, m^{\prime}}=\pi$, it does not have its real part because it is expressed as $j 2 a \sin \left\{2 \pi f_{D}\left(\cos \theta_{k l, m}\right) t\right\}$. Thus, the $y$-axis symmetric arrangement of scattering points may cause instability on statistical fading properties. To avoid such phenomena, the following condition on arrangement of scattering points should be satisfied

$$
\theta_{k l, m} \neq \pi-\theta_{k l, m^{\prime}} \quad \text { for } 1 \leq m, m^{\prime} \leq M .
$$

That is, scattering points should not be arranged symmetrically to the $y$-axis. We define the above condition as the intra-ring condition.

\section{Inter-Ring Condition}

When using multiple scattering rings, arrangement of scattering points across the rings should be considered. At first, we assume a time-varying $N_{\text {rx }} \times N_{\text {tx }}$ MIMO channel $\boldsymbol{H}(t)$ based on $N_{\mathrm{tx}} N_{\mathrm{rx}}$ scattering rings, where all the rings have the same scattering-point structure. The channel $\boldsymbol{H}(t)$ can be decomposed into $M$ channel components
$\boldsymbol{H}_{1}(t), \ldots, \boldsymbol{H}_{M}(t)$, where the $m$ th component $\boldsymbol{H}_{m}(t)$ is composed of the $m$ th scattered waves in all the $N_{\mathrm{tx}} N_{\mathrm{rx}}$ rings, i.e., $h_{k l, m}(t)$ for $k=1, \ldots, N_{\mathrm{rx}}$ and $l=1, \ldots, N_{\mathrm{tx}}$. In $\boldsymbol{H}_{m}(t)$, all the angles of arrival of wave components are the same, i.e., $\theta_{k l, m}=\theta_{m}$ regardless of antenna indices $k$ and $l$, due to the same arrangement. Hence, $\boldsymbol{H}_{m}(t)$ can be expressed as

$$
\boldsymbol{H}_{m}(t)=a e^{j 2 \pi f_{D}\left(\cos \theta_{m}\right) t} \boldsymbol{\Phi}_{m},
$$

where $\boldsymbol{\Phi}_{m}$ represents an $N_{\mathrm{rx}} \times N_{\mathrm{tx}}$ initial phase matrix in which an element of the $k$ th row and $l$ th column is $\phi_{k l, m}$.

The above equation implies that regularity of the matrix $\boldsymbol{H}_{m}(t)$ depends on the given initial phase set $\boldsymbol{\Phi}_{m}$. In the case of $\phi_{11, m}=\phi_{12, m}=\cdots=\phi_{N_{\mathrm{rx}} N_{\mathrm{tx}}, m}$, it is obvious that the matrix is singular, i.e., $\operatorname{rank}\left[\boldsymbol{H}_{m}(t)\right]=1$, regardless of time. Of course this is an over-simplified example, and the actual channel matrix $\boldsymbol{H}(t)$ is superposed by $M$ channel components so that its regularity will be maintained. It is supposed, however, that such singular matrix components may cause an unstable property of $\boldsymbol{H}(t)$. The simplest way to avoid this is to satisfy the following condition on arrangement of scattering points across rings defined as the inter-ring condition

$$
\theta_{k l, m} \neq \theta_{k^{\prime} l^{\prime}, m^{\prime}} \quad \text { for }\left\{\begin{array}{l}
1 \leq k, k^{\prime} \leq N_{\mathrm{rx}} \\
1 \leq l, l^{\prime} \leq N_{\mathrm{tx}} \\
1 \leq m, m^{\prime} \leq M
\end{array},\right.
$$

except $m=m^{\prime}$ in the case of $k=k^{\prime}$ and $l=l^{\prime}$.

\section{Numerical Analysis}

We simulated time-varying channels based on Jakes' model for single-input single-output (SISO) and $2 \times 2$ MIMO cases to evaluate the intra- and inter-ring conditions. We set $M=12$ for each scattering ring and prepared 10,000 initial phase sets randomly given ${ }^{\dagger}$. For each initial phase set, we generated time-varying channels based on (1) and captured 1,000,000 snapshots to obtain its cumulative distribution function (CDF) of amplitudes and eigenvalues for SISO and MIMO cases, respectively.

Figure 3 demonstrates three examples for SISO channels yielded by different initial phase sets, where all the three Jakes' rings had the same scattering-point structure without the intra-ring condition, i.e., $y$-axis symmetric structure. Figure 3(a) shows CDFs of amplitudes for the three cases. For comparison, we also show the performance obtained by complex Gaussian random process. It can be seen in Fig. 3(a) that the three CDFs do not agree with that of the Gaussian random process case and depend on given initial phases while they all show the Rayleigh distribution characteristic, i.e., the increase of one order of magnitude with an amplitude increment of $10 \mathrm{~dB}$ in their linear parts. Its impact on bit error rate (BER) performance is shown in Fig. 3(b), where QPSK modulation was used. In the BER performance, difference similar to that in CDFs can be seen. This implies that the variation in CDFs of channels directly

${ }^{\dagger}$ In [5], Jakes showed that Rayleigh fading can be simulated with $M=8$. 
appears in transmission performance. To evaluate the fluctuation, we observed an additional CDF of $10 \%$ values (hereinafter we refer to it as $10 \%$-value $\mathrm{CDF}$ ) for each scatteringpoint arrangement. Also, we measured a value spread $\Delta$ defined as difference between $1 \%$ and $99 \%$ values in the $10 \%$-value CDF. The value spread $\Delta$ corresponds to possible fluctuation range of transmission performance over timevarying channels generated by Jakes' model with different initial phase sets.

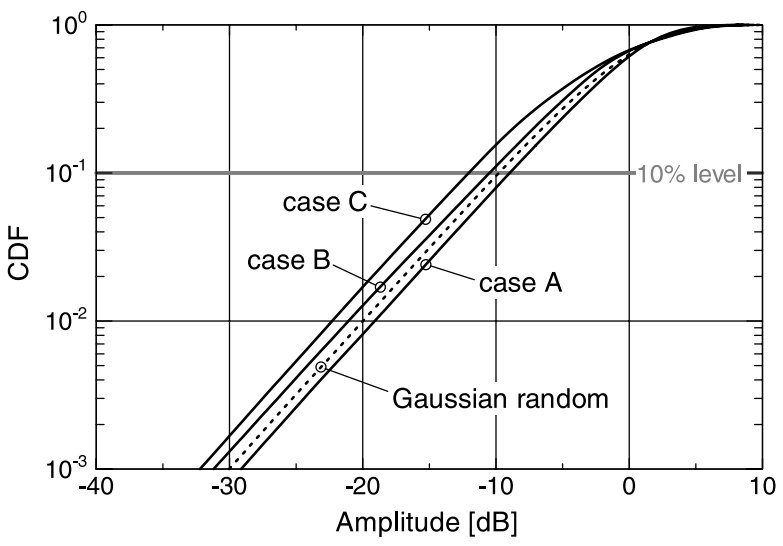

(a) $\mathrm{CDF}$ of amplitudes

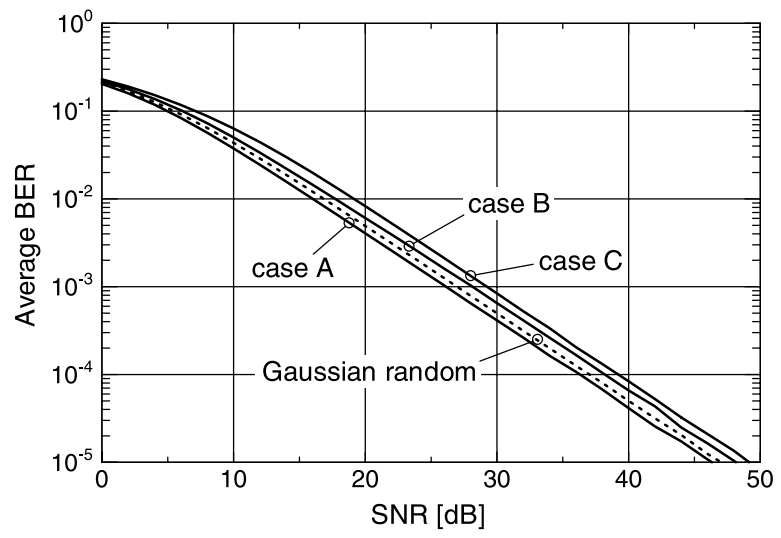

(b) BER performance

Fig. 3 Examples of SISO channels based on Jakes' model.

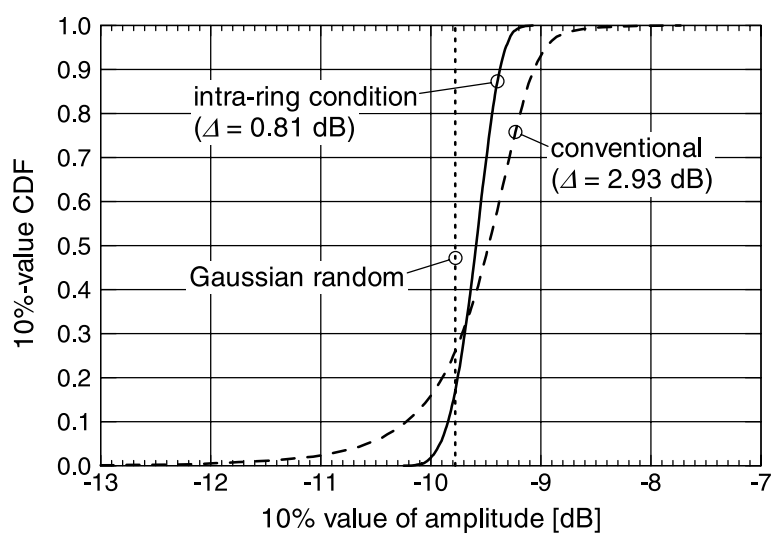

Fig. $4 \quad 10 \%$-value CDFs of amplitudes for SISO cases.
We first evaluate the intra-ring condition for SISO channels by using $10 \%$-value CDFs of the amplitudes and their value spreads shown in Fig. 4. Here, "conventional" denotes a case of $y$-axis symmetric arrangement of scattering points. As a reference, we also show a $10 \%$ value for a Gaussian random process case. It is clear that the conventional arrangement yields various fading states depending on the initial phase setting. On the other hand, the arrangement under the intra-ring condition provides more stable fading properties compared to the conventional arrangement. The value spread is effectively reduced to $0.81 \mathrm{~dB}$ from $2.93 \mathrm{~dB}$ with the intra-ring condition. We confirmed that $M>30$ is necessary for the conventional arrangement to achieve the same stability as the arrangement under the intra-ring condition with $M=12$, i.e., to reduce the value spread $\Delta$ to $0.81 \mathrm{~dB}$

Next, we evaluate the inter-ring condition. Figure 5 shows 10\%-value CDFs of the first (maximum) and second (minimum) eigenvalues for $2 \times 2$ MIMO channels, where both arrangement types, "inter-ring condition" and "conventional," are constrained by the intra-ring condition. We can see from the CDFs for the conventional arrangement that a common arrangement over all the scattering rings causes properties dependent upon the initial phase setting even through under the intra-ring condition. Furthermore, the difference is larger for the second eigenvalues. In con-

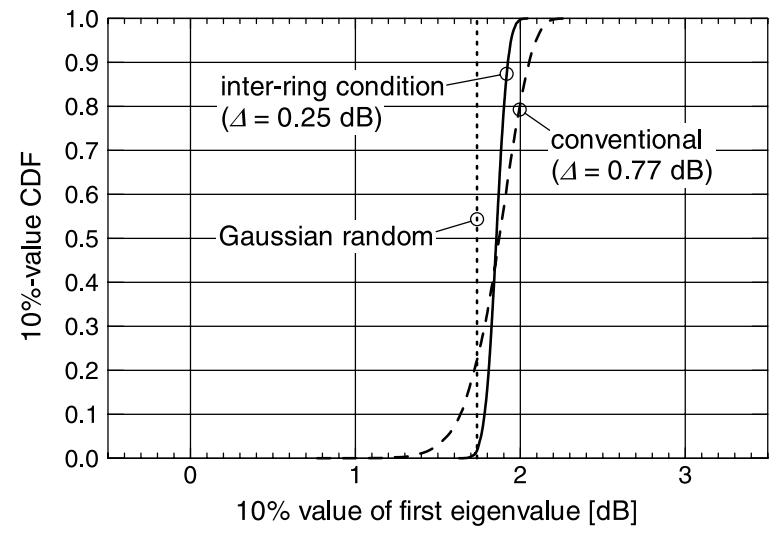

(a) First eigenvalue

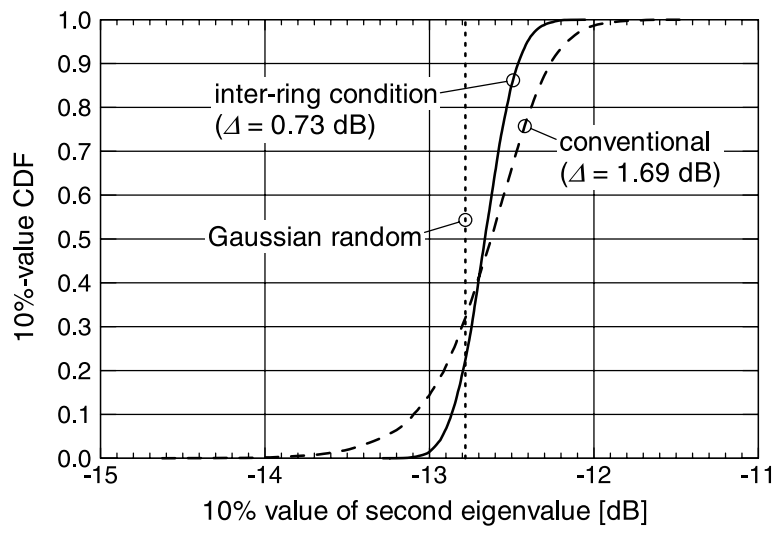

(b) Second eigenvalue

Fig. $510 \%$-value CDFs of eigenvalues for $2 \times 2$ MIMO cases. 


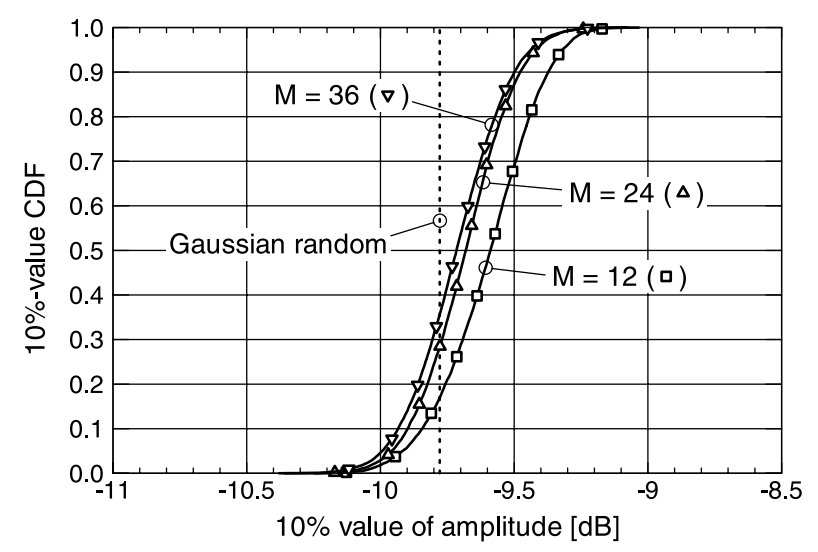

Fig. $610 \%$-value CDFs of SISO channels under the intra-ring condition in the cases of $M=12,24$, and 36 .

trast, the arrangement under the inter-ring condition gives much more stable eigenvalue properties. With the condition, the value spread for the first eigenvalues is reduced to $0.25 \mathrm{~dB}$ from $0.77 \mathrm{~dB}$, and that for the second eigenvalues is reduced to $0.73 \mathrm{~dB}$ from $1.69 \mathrm{~dB}$.

It is seen in Figs. 4 and 5 that $10 \%$-value CDFs of Jakes' rings are mostly located in higher amplitude/eigenvalue regions than those of Gaussian random process cases. We can achieve a random process similar to the Gaussian random case if we have a sufficiently large number of scattering points, i.e., $M \rightarrow \infty$. However, using Jakes' model implies angular sampling of a uniformly distributed angular spectrum by finite scattering points, which may result in an insufficient random nature. This is therefore a specific issue of Jakes' model with a finite number of scatterers. It is expected that the issue may be mitigated by increasing the number of scattering points $M$. Figure 6 demonstrates $10 \%$-value CDFs of SISO channels under the intra-ring condition in the cases of $M=12,24$, and 36. Although locating many scatterers is not desirable for us as mentioned in Introduction, it is proved from Fig. 6 that CDFs of Jakes' model approach the Gaussian random case as $M$ increases. Consequently, the phenomenon mentioned above is not clearly seen in the case of $M=36$.

\section{Conclusions}

We have established simple and effective conditions on scattering-point arrangement in Jakes' model for stable fading simulations. We confirmed that, for a single scattering ring, the intra-ring condition is effective for obtaining stable fading property in the aspect of statistics regardless of the initial phase setting. Moreover, it was shown that arrangement under the inter-ring condition provides fading properties robust to the given initial phases in a multiple-ring case such as $2 \times 2$ MIMO channels. It should be noted that the proposed inter-ring condition can be effective not only for MIMO flat fading channels but also for other fading channels, e.g., SISO channels.

\section{Acknowledgement}

This work was supported in part by a Research Fellowship for Young Scientists from the Japan Society for the Promotion of Science.

\section{References}

[1] G.J. Foschini and M.J. Gans, "On limits of wireless communications in a fading environment when using multiple antennas," Wirel. Pers. Commun., vol.6, no.3, pp.311-335, 1998.

[2] I.E. Telatar, "Capacity of multi-antenna Gaussian channels," Euro. Trans. Telecommun., vol.1, no.6, pp.585-595, Nov./Dec. 1999.

[3] E. Biglieri, R. Calderbank, A. Constantinides, A. Goldsmith, A. Paulraj, and H.V. Poor, MIMO Wireless Communications, Cambridge University Press, New York, 2007.

[4] A. Ghosh, D.R. Wolter, J.G. Andrews, and R. Chen, "Broadband wireless access with WiMax/802.16: Current performance benchmarks and future potential," IEEE Commun. Mag., vol.43, no.2, pp.129136, Feb. 2005.

[5] W.C. Jakes, Jr., Microwave Mobile Communications, Wiley, New York, 1974.

[6] P. Dent, G.E. Bottomley, and T. Croft, "Jakes fading model revisited," Electron. Lett., vol.29, no.13, pp.1162-1163, June 1993. 